\title{
Characteristics of Psychiatric Patient's Use of One Emergency Department and Crisis Services and Comparison of Models of Care for Patients with Borderline Personality Disorder in the Frequency of Use of Emergency Services
}

Ramamohan Veluri ( $\nabla$ rveluri@outlook.com )

Stacey Roles

Northern Ontario School of Medicine

Roberta Heale

Laurentian University

Vicky P. K. H. Nguyen

Northern Ontario School of Medicine

M. Elizabeth Renouf

University of Waterloo

Research article

Keywords: urgent care, borderline personality disorder, accessibility

Posted Date: November 7th, 2019

DOl: https://doi.org/10.21203/rs.2.16945/v1

License: (c) (1) This work is licensed under a Creative Commons Attribution 4.0 International License.

Read Full License 


\section{Abstract}

Background: The cost of urgent mental health care services is high, both to the system as well as the individual and strategies that prevent these visits are paramount. Frequent users of the emergency department (ED) are a small percentage of ED patients but account for a large percent of visits. In particular, studies show that those patients with borderline personality disorder commonly present to urgent care. Given the rising number of ED visits and the corresponding cost to the health care system, it is important to explore strategies for avoidable or preventable visits to the ED, coupled with directing resources that ensure access to appropriate quality care.

Methods: This research was a prospective chart review of the population of frequent users diagnosed with psychiatric illness in the (ED) and crisis (CRI) services at one hospital. Detailed analysis revealed characteristics of the patients who presented to the ED and CRI with a mental health diagnosis. Additional analysis of the subgroup of these patients with borderline personality disorder and/or self-harming behaviour was completed to determine the impact of the model of psychiatric care on subsequent crisis or ED visits in the year after the index visit.

Results: The majority of patients presenting for mental health issues did so once. The mean number of subsequent presentations to the ED was 5.00 demonstrating that a small number of patients presented many times. Male patients were trending toward significance for number of ED and CRI visits. Patients with borderline personality disorder and/or self-harming behaviours in a model of care that offered increased access to services were less likely to visit the ED and CRI.

Conclusions: Unstable or complex patients with psychiatric issues often present at the ED and CRI. Accessibility to community care services has the potential to reduce the number of ED and CRI visits and is a critical factor when considering this less stable group.

\section{Background}

This project is a prospective chart review aimed at characterizing the population of frequent users diagnosed with psychiatric illness of the Crisis Department and Emergency Department (ED) services at the hospital in Sudbury, Ontario, Canada. Detailed analysis was also completed on the subgroup of these patients with borderline personality disorder (BPD) and/or self-harming behaviours to determine the impact of two models of psychiatric care on subsequent crisis or ED visits in the year after the index visit.

Canadian hospitals spent close to \$2 billion in 2005-2006 on ED care. In Ontario, estimates by the Canadian Institute for Health Information $(\mathrm{ClHI})$ indicate that the average amount spent on each visit to be $\$ 148$ [1]. More recent $\mathrm{ClHI}$ data also suggest that the annual number of ED visits in Canada will increase from 15 million per year in 2013 to over 21 million in 2043 (or an increase by 40\%) [2]. Given the rising number of ED visits and the corresponding cost to the health care system, it is important to explore strategies for avoidable or preventable visits to the ED, coupled with directing resources that ensure access to appropriate quality care. 
One study demonstrated that approximately $35 \%$ of all ED visits are related to minor physical injuries with normal physiology. Of this patient population, an estimated $44 \%$ had a psychiatric diagnosis at the time of the study or in the past [3]. Of interest also, were those patients who present to the ED more than once with the same issue. Frequent users of the ED tended to make up a small percentage of ED patients but accounted for a large percent of visits, $3 \%$ and $18 \%$, respectively according to one study [4] and $5 \%$ and $27 \%$, respectively, in another study [5]. Predictors of frequent ED use include limited social support, homelessness, cocaine positive-toxicology screen, documented personality disorder/traits, self-reported alcohol and substance use, and psychotic illness/antipsychotic medication use. [4,6,7,8]. In particular, studies show that those patients with borderline personality disorder (BPD) commonly present to urgent care. For example, the results of a four -year study in a tertiary hospital in Spain demonstrated that BPD was the diagnosis in $9 \%$ of the visits. The authors concluded that the vast majority of these visits could have been avoided or prevented if the patients had been able to access appropriate care in the community [9].

Reasons for psychiatric patients' use of the ED and crisis services vary, however, many do so because they are unable to access care in the community (10). In addition, urgent care centres are most often do not have the appropriate resources to care for patients with complex mental health concerns, such as those with BPD (10).

BPD is a complex and multidimensional disorder. Patients present with a wide range of comorbidities and with traits of affective instability and impulsivity, including self-harming behaviours. Although overall prognosis is good, many people with BPD will require lengthy treatment (11). It's common for patients to stop treatment when they have improved. If symptoms worsen, resources may no longer be in place, with the ED and Crisis as the most available options. Treatment for BPD helps to stabilize patients so access to therapy should be a priority (11).

\section{Model of Care}

It is well documented that the model of care for patients with complex psychiatric presentations impacts their use of ED. In one study, a transitional psychiatric clinic (TPC) bridged patents after an ED visit and showed an increase attendance at aftercare along with lengthened time without ED presentation [12]. A full-service partnership program in the community is the approach taken by the California Institute for Mental Health with the philosophy of doing "whatever it takes" to help individuals to recover and achieve wellness. This program is patient-centred with services focused on individual patient needs. One outcome is reduction in urgent care [13]. In another example, a model of shared care between psychiatrists and family doctors in northern Ontario, Canada, significantly reduced wait times for care for patients with psychiatric disorders (14). Finally, studies about the care of patients with BPD demonstrated that the availability of a variety of treatment options tailored to the acuity and level of complexity of their presentations had the best outcomes (11). These studies showed that directing resources to address mental and physical health needs of this population of patients in a timely manner in the community, 
helped to reduce the burden of mental illness and thereby the number of crisis events and visits to the ED.

There are two unique models of psychiatric care in Sudbury, Ontario. In the first traditional care model, patients with BPD or self-harming behaviours presenting at the ED, or crisis are referred to see one in a list of psychiatrists with practices in the city, or to their own psychiatrist if they are already receiving care. The patients are given a follow up appointment with the psychiatrist when available.

In the second model, the office manager in one practice knows the patients well and assists in triaging need and appointment times with their psychiatrist. In this second model of care there are also transdiagnostic groups by the psychiatrist to provide timely treatment and quicker ability to make medication changes, assist with work-life stress and facilitate admission to hospital if necessary. When necessary, the patient was counseled on phone. This model gave the patients the feeling that their psychiatrist is available always.

\section{Study}

The aims of this study were to first identify the characteristics of frequent crisis and ED users, in particular those with BPD and self -harm. Secondly, service use was analyzed and compared to subsequent $E D$ and crisis visits of those in one unique model of psychiatric care to those receiving traditional care. Findings from this study have the potential to inform the design of further studies and target, evidence-informed clinical interventions aimed at reducing crisis events and unnecessary visits to the ED. By determining the level of burden of mental health of patients who frequently use crisis services and the ED, and by further examining the effectiveness of various models of care on these patients, we will be positioned to make recommendations for optimal treatment options.

\section{Methods}

\section{Setting}

Health Sciences North (HSN) is the largest hospital in northern Ontario, Canada and the only tertiary care hospital within an area encompassing several hundred kilometers. It offers urgent care services for people with mental health issues through the ED and the affiliated Crisis Department (CRI). There are many mental health care resources in the area, but they are mostly clustered in the city of Sudbury proper. The catchment area for psychiatric services encompasses the entire region which includes a large rural area and many small towns. These barriers add to the importance of exploring the model of psychiatric care (15).

A prospective chart review was conducted to collect data on patients aged 18-59 attending ED and CRI visits to HSN in 2015. Variables included psychiatric diagnoses, dates and reasons for ED visits including crisis events, use of crisis services and number of total admissions to psychiatry in-patient care. In addition, data were collected about presentations to community mental services including physician 
follow-ups following crisis events and the frequency of visits to the ED before and after the initial ED visit in 2015.

Data were obtained from HSN charts which included visits to ED and CRI as well as psychiatric outpatient clinic (POC) and outpatient mental health clinics. Data from the charts were also obtained from two standardized patient assessments tools. The tools are completed by regulated clinical staff, other than a psychiatrist, when a patient presents to services. The first is the Common Data Set Mental Health (CDS) assessment. The CDS is used for collecting administrative and clinical data for community mental health patients with some information on outcomes (16).

The second assessment tool is the inter Resident Assessment Instrument Mental Health (RAl) Assessment System. The RAl is a comprehensive standardized instrument for evaluating the needs, strengths and preferences of adults with mental illness in inpatient psychiatric settings and is completed by nurses from the inpatient units (17). These are provincially mandated assessment tools to be completed for every patient in the province of Ontario. These tools also provide information about where the patient was seen and by whom. For example, a POC is a visit with a psychiatrist whereas the CDS, if completed, represents a visit to an outpatient centre including the emergency department and crisis services and RAI represents a psychiatric inpatient admission.

\section{Results}

There was a total of 73,116 ED visits to the target hospital in $2015,40,055$ of which were for patients between the ages of 18 and 59. Of this latter group, 2,879 presented with mental health issues. In particular, 220 ED visits were related to BPD or self -harming behaviour.

The remaining highest mental health presentations to the ED include:

932 - Mental and behavioural disorders due to psychoactive substance use

452 - Depressive episode, unspecified

403 - Anxiety disorder, unspecified

304 - Acute stress reaction

123 - Unspecified nonorganic psychosis

97 - Schizophrenia, unspecified 
Of the 220 BPD and self-harm presentations, there were 192 unique patients. Of these, 129 patients presented once to ED and did not have a follow up ED or crisis presentation. Sixty-three of the 192 unique patients (32.8\%) accounted for an additional 66 ED visits within one year from their index visit and 157 visits to crisis. These 63 patients accounted for 286 total visits (including index) to ED/crisis within a one-year time frame. This clearly suggests that there were differences in the level of acuity of this group of patients with BPD.

Many patients had evidence of a POC, a completed CDS, or RAl, either individually or in combination with one or both of the other options (see Table 1). Analysis of variance showed that the differences in $E D+C R I$ counts for these groups is significantly different $(p=0.01)$. To determine which groups were significantly different from each other a Tukey Multiple Pairwise Comparison test found that subjects receiving all care, POC+CDS+RAI 83/192 (43\%), have a significantly higher count of ED+CRI than subjects using none $(p=0.03)$. These subjects also have the highest use of ED+CRI. Not surprisingly, these findings suggest that the clients who utilize all services more often are the same patients who also utilized the ED and CRI most often.

\section{Table 1. Frequency of POC visits and Completion of CDS or RAI}

\begin{tabular}{lcll} 
Group & N & Mean \# ED+CRI & SD \\
\hline POC only & 10 & 0.10 & 0.32 \\
\hline CDS only & 12 & 0.58 & 1.00 \\
\hline RAl only & 13 & 0.15 & 0.38 \\
\hline CDS + POC & 13 & 2.23 & 2.28 \\
\hline RAI + POC & 17 & 0.24 & 0.44 \\
\hline CDS + RAI & 13 & 0.92 & 1.04 \\
\hline CDS + RAI + POC & 83 & 4.66 & 10.00 \\
\hline NONE & 31 & 0.03 & 0.18
\end{tabular}

Overall, the mean number of ED+CRI visits for all subjects in the study period (not including the index visit) was 2.3. The minimum was 0 and the maximum 70 .

There were a large number of subjects with no visits to either ED or CRI aside from the index visit. Fiftyfour percent (104/192) of subjects had only one visit to ED or CRI in the study period before or after the index visit. Considering ED and CRI separately, $80 \%$ of patients do not have additional ED visits in the year before or the year after the index visit and $59 \%$ of subjects do not visit Crisis Services in the years before or after the index visit. Considering only the subjects who had at least one additional visit to ED or 
$\mathrm{CRI}$, the mean number of visits in the study period was 5.0. Figure 1 shows the number of ED+CRI visits grouped by whether the subject had a CDS, a RAI or was seen in POC.

\section{Figure 1: Number of ED+CRI visits grouped by whether the subject had a CDS, a RAI and was seen in POC.}

A generalized linear model with a negative binomial distribution was found to be the most appropriate for analyzing factors affecting the number of ED + CRI visits. The response variable, the total number of $E D+C R I$ visits per patient in the year before and after the index visit, is represented by a count of the number of visits for each study subject. Other variables in the model were as follows: The number of POC visits, indicating the number of times the subject was seen by a psychiatrist during the study period. CDS and RAI are indicators of whether a CDS or RAl assessment was done during the study period. For those 121 subjects who had a CDS assessment, 15 of them were done on the same day as the index visit, 72 were done after the visit date and 34 were before after the visit date. A total of 126 subjects had an RAl assessment done, 3 of which were done on the same day as the visit date, 15 were done before the visit data and 108 were done afterwards. Also included in the model were subject age and sex, and whether the index visit indicated self -harm or BPD.

The model was selected based on a stepwise method using the Akaike Information Criterion which is used to determine the best model fit. The best fitting model found that the following variables were significant at $p<0.05$ : the number of POC visits, whether a CDS assessment was done, and whether an RAl assessment was done. Sex was trending toward significance and it helped improve model fit therefore it was kept in the model.

Table 2. Final Model Output

\begin{tabular}{llll} 
Coefficients & Estimate & Std. Error & $\operatorname{Pr}(>|z|)$ \\
\hline Intercept & -2.88891 & 0.44977 & $1.34 \mathrm{e}-10$ \\
\hline Total POC & 0.03960 & 0.01144 & 0.00054 \\
\hline CDS any & 2.85205 & 0.42236 & $1.45 \mathrm{e}-11$ \\
\hline RAl any & 0.78554 & 0.32879 & 0.01688 \\
\hline sex: Male & 0.47763 & $\mathrm{~A} 0.26066$ & 0.06690
\end{tabular}


All variables in the model except for sex are significant at a $p$ value $<0.02$. However, sex has a $p$ value of approximately 0.07 and is trending toward significance. In addition, male subjects have a higher expected count of ED+CRI visits $(p=0.07)$.

Below we present a table showing the expected counts of ED+CRI. We stop at $20 \mathrm{POC}$ visits since there were only 6 subjects with greater than 20 visits ( 2 male, 4 female).

\section{Table 3. Expected Counts of ED + CRI Visits}

\begin{tabular}{lllll} 
\# POC visits & CDS completed & RAl completed & Sex & Expected \# of visits \\
\hline None & No & No & Female & 0.056 \\
\hline None & Yes & No & Female & 0.964 \\
\hline None & No & Yes & Female & 0.122 \\
\hline None & No & No & Male & 0.090 \\
\hline 1 & No & No & Female & 0.058 \\
\hline 10 & No & No & Female & 0.083 \\
\hline 20 & No & No & Female & 0.183
\end{tabular}

These results indicate that the subjects who do not require a POC, RAI and CDS present the least often to $E D+C R I$. Subjects requiring 'POC only' with less than 20 POC visits in the study period present to ED and CRI less often than the subjects who also have a CDS or RAI assessment. Having a CDS, or an RAl, or being male increases the expected count of ED + CRI visits. As the number of POC visits increases, so does the expected count of ED + CRI visits. Analysis shows that as total POC visits increase, so do predicted ED+CRI visits. Self -harm and BPD at index visit were not found to be significant predictors of the number of ED+CRI visits during the study period.

\section{Subgroup analysis: Subset of those with any ED+CRI visits}

A logistic regression on factors affecting a decrease in $E D+C R I$ visits year over year was run. Although a large portion of the subjects had no ED or CRI visits before or after the index visit, it is of clinical interest to examine characteristics of those subjects with a positive count in $E D+C R I$, specifically whether the number of visits increased or decreased from year 1 to year 2 in the study period. 
A total of $34 / 88$ subjects (38.6\%) of these subjects had a year over year decrease in the number of ED+CRI visits, $11 / 88$ (12.5\%) experienced the same number of visits, and 43/88 (48.9\%) experienced an increase in the number of visits from year 1 to year 2 . The only significant variable found to contribute to a decrease in the number of ED+CRI visits was whether a CDS assessment was done in the first year of the study (i.e., prior to the index visit). The log odds of having a decrease in visits in year 2 if a CDS assessment is done before the index visit is 1.48 with a $95 \%$ confidence interval $(0.54,2.47)$. This means that a CDS assessment before the index visit significantly increased the odds of having a decrease in the total number of ED+CRI visits in the second year of the study $(p=0.0025)$.

\section{Psychiatric Outpatient Clinic: Standard vs Unique Model of Care}

We conducted several analyses to determine if there were differences between the ED and CRI visits of patients receiving traditional care or care in the unique model. There were 123 subjects $(64.1 \%)$ seen in POC either before or after the index visit. Of these, 70 subjects had a POC visit prior to the index visit, 117 had a POC visit after the index visit and 64 subjects had a POC visit both before and after the index visit. For this group of 64 subjects, we calculated the overall mean time between the last POC visit before the index and the next POC visit after the index visit as 104 days.

For the first model of care, for everyone with a POC visit prior to the index (70 subjects), the mean time between the POC visit prior to the index visit and the date of the index visit itself was 76 days. For everyone with a POC visit following the index (117 subjects), the mean time between the POC visit after the index visit and the date of the next POC visit was 77 days.

Six subjects were seen in the unique practice model, with a setup meant to allow more accessibility. Of these six subjects, five were seen in POC both before and after the index visit. The mean time between the last POC visit before the index visit and the next POC visit after the index visit for these five subjects was 44 days, compared to the much larger mean for the other 59 subjects in the traditional practice model at 109 days. With the caveat that the sample size for the accessible practice model is small $(n=5$ vs $n=59$ ), we carried out Welch's two sample $t$ test for comparing means with unequal variances and found that the accessible practice model subjects have a significantly shorter time between POC visits $(p=0.0001)$.

We used Fisher's Exact Test for Count Data to analyse differences in the number of ED+CRI visits, by practice model. While not reaching statistical significance $(p>0.05)$ the findings are still clinically relevant. Again, with our small sample size, this result should be interpreted with caution, however, clinically, in this POC subset, $4 / 6(66.7 \%)$ of subjects in the accessible practice model had $0 \mathrm{ED}+\mathrm{CRI}$ visits while in the traditional practice model, $45 / 117$ (38.5\%) of subjects had $0 \mathrm{ED}+\mathrm{CRI}$ visits.

For the unique practice model, the mean time between the POC visit prior to the index visit and the date of the index visit itself was 21 days ( 5 subjects). For everyone with a POC visit following the index (6 subjects), the mean time between the POC visit after the index visit and the date of the next POC visit was 24 days. For the traditional practice model, the mean time between the POC visit prior to the index visit 
and the date of the index visit itself was 80 days (65 subjects). For everyone with a POC visit following the index (111 subjects), the mean time between the POC visit after the index visit and the date of the next POC visit was also 80 days.

\section{Discussion}

This study adds to the body of knowledge about characteristics of patients with mental health issues who present to the ED and CRI. The majority of patients had only one visit to ED or CRI in the study period and of those who had at least one additional visit to ED or CRI, the mean number of visits in the study period was 5.00. A small number of people had a high number of ED and CRI visits suggesting either lack of access to care in the community, complex cases resistant to treatment, or a combination of both. These findings are similar to previous research $(3,4,5,9)$. Of interest, and worthy of future research, is the finding that the number of ED and CRI visits for male patients in this study was trending toward significance.

Additional analysis with a subgroup of patients with BPD and self-harming behaviours demonstrates that a model of care with increased accessibility to psychiatric and other services has the potential to mitigate subsequent $E D$ and $C R I$ visits. The small numbers in the comparison of models of care offer some interesting trends, but cannot be generalizable to a larger group. However, it's important to also note that the data collected for the unique model do not reflect the total number of patients with BPD or self -harm in this model of care who did not present to the ED or Crisis, which is 17. In addition, the HSN charts did not fully capture the 6 patient's presentations to group care within the model, which occurred twice per month in the research time frame. More comprehensive evaluation of models of care is warranted.

\section{Conclusions}

The cost of urgent mental health care services (ED and CRI) is high, both to the system as well as the individual and strategies that prevent these visits are paramount, particularly for those with complex and acute presentations. The findings of this study confirm the majority of patients with BPD and self-harm have one or few visits to the ED and CRI and use follow up services less often. The focus should be on the remainder, those who utilize all services more frequently. Accessibility to community care services has the potential to reduce the number of ED and CRI visits and is a critical factor when considering this less stable group. Although numbers are small, the unique model of care shows promise in achieving this objective. Future research should continue to be focused on the outcomes related to model of practice.

\section{Acronyms}

Borderline personality disorder (BPD) 
Canadian Institute for Health Information (CIHI)

Common Data Set Mental Health (CDS)

Crisis Department (CRI)

Emergency Department: (ED)

Health Sciences North (HSN)

psychiatric outpatient clinic (POC)

Resident Assessment Instrument Mental Health (RAI)

\section{References}

1. Dawson H, Zinck G. ClHI Survey: ED spending in Canada: a focus on the cost of patients waiting for access to an in-patient bed in Ontario. Healthcare quarterly (Toronto, Ont.) 2009, 12:25-28.

2. Canadian Foundation for Healthcare Improvement (Ed): The Impact on Emergency Department Utilization of the CFHI Healthcare Collaborations and Initiatives 2013, May.

3.Richmond T S, Hollander J E, Ackerson T H, Robinson K, Gracias V, Shults J, Amsterdam J. Psychiatric disorders in patients presenting to the Emergency Department for minor injury. Nursing Research 2007, 56:275-282.

4. Vandyk A D, VanDenKerkhof E G, Graham I D, Harrison M B. Profiling frequent presenters to the emergency department for mental health complaints: socio-demographic, clinical, and service use characteristics. Archives of psychiatric nursing 2014, 28:420-425.

5. Sullivan P F, Bulik C M, Forman SD, Mezzich J E. Characteristics of repeat users of a psychiatric emergency service. Hospital \& community psychiatry 1993, 44:376-380.

6. Chang G, Weiss A P, Orav E J, Rauch S L. Predictors of frequent emergency department use among patients with psychiatric illness. General hospital psychiatry 2014, 36:716-720.

7. Boyer L, Dassa D, Belzeaux R, Henry J, Samuelian J, Baumstarck-Barrau K, Lancon C. Frequent visits to a French psychiatric emergency service: diagnostic variability in psychotic disorders. Psychiatric Services (Washington, D.C.) 2011, 62:966-970.

8. Chaput, Yves J A, Lebel M. An examination of the temporal and geographical patterns of psychiatric emergency service use by multiple visit patients as a means for their early detection. BMC Psychiatry 2007, 7:60.

9. Pascual J C, Córcoles D, Castaño J, Ginés JM, Gurrea A, Martín-Santos R, Garcia-Ribera C, Pérez V, Bulbena A. Hospitalization and pharmacotherapy for borderline personality disorder in a psychiatric 
emergency service. Psychiatric Services (Washington, D.C.) 2007, 58:1199-1204.

10. Zun, L. Pitfalls in the care of psychiatric patient in the emergency department. The Journal of Emergency Medicine 2012, 43:5.

11. Paris J. Stepped care: An alternative to routine extended treatments for patients with borderline personality disorder. Psychiatric Services 2013, 64:10

12. McCullum smith C, Clark B, Blair C, Cropsey K, Shelton R. Rapid follow-up for patients after psychiatric crisis. Community mental health journal 2015, 51:139-144.

13. Brown T T, Chung J, Choi S, Scheffler R, Adams N. The impact of California's full-service partnership program on mental health-related emergency department visits. Psychiatric Services (Washington, D.C.) $2012,63: 802-807$.

14. Haggarty J M, Jarva J A, Cernovsky Z, Karioja K, Martin, L. Wait time impact of co-located primary care mental health services: the effect of adding collaborative care in northern Ontario. Canadian Journal of Psychiatry 2012, 57:1.

15. Ontario Ministry of Health and Long-Term Care. Health Services in Your Community. Hospital Locations and Classifications by LHIN.

http://www.health.gov.on.ca/en/common/system/services/hosp/northeast.aspx_Accessed Sept 2019.

16. Ontario. Common Data Set / Mental Health (CDS/MH). https://www.ontario.ca/data/common-dataset-mental-health-cds-mh Accessed Sept 2019.

17. InterRAI. Mental Health for Inpatient Psychiatry. http://www.interrai.org/mental-health.html Accessed Sept 2019.

\section{Declarations}

\section{Ethics approval and consent to participate}

Ethics approval was received from Health Sciences North Research Ethics Board project number 17101 and Laurentian University Research Ethics Board file number 6012449 to collect a dataset from patient files from HSN.

Consent for publication: Not applicable

\section{Availability of data and materials}

The dataset generated and analysed during the current study is not publicly available as per privacy laws in Canada and the ethics committee that approved this study.

Competing interests: None

\section{Funding}


Funding for this research project was received from the Northern Ontario Academic Medicine Association. The funding body had no role in the design, collection, analysis or interpretation of the data related to the study.

\section{Authors' contributions}

Dr. R. Veluri was involved in each stage of the research process and added to the data and description of this study from his practice and assisted with the interpretation of results of this study. Stacey Roles and Roberta Heale were involved in each stage of the research process including drafting of the article. Dr. Nguyen was involved in the development of the research proposal.R. Elizabeth Renouf was the statistician for this project and wrote the analysis section of this article.

Acknowledgements: Not applicable

\section{Figures}




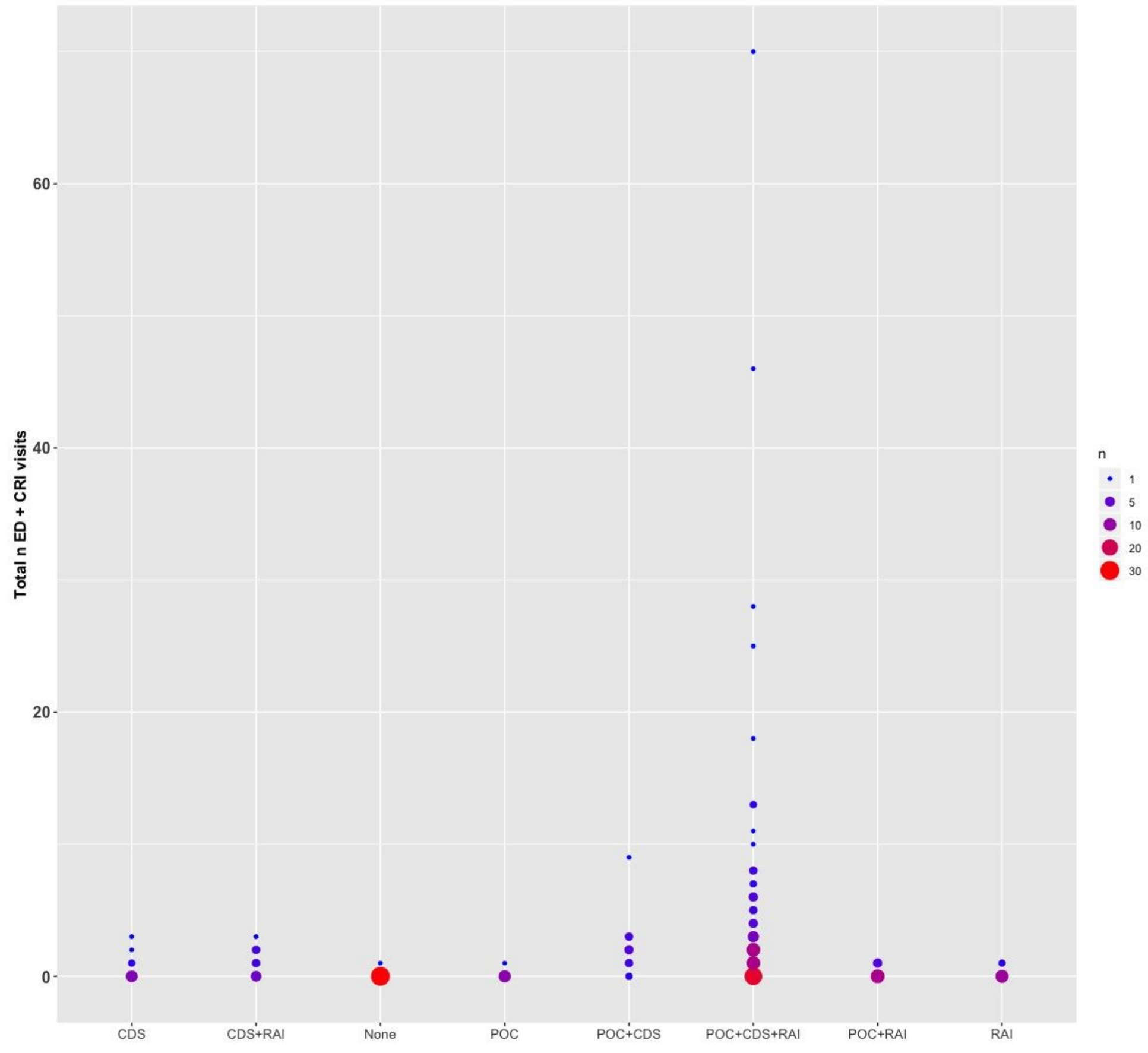

Figure 1

Number of ED+CRI visits grouped by whether the subject had a CDS, a RAI and was seen in POC. 